\title{
Association Between Insomnia and Migraine Risk: A Case-Control and Bidirectional Mendelian Randomization Study
}

\author{
Shujuan Chu' \\ Zhilin Wu' \\ Zhouyang $\mathrm{Wu}^{\prime}$ \\ Jing $W u^{\prime}$ \\ Yue Qian ${ }^{2}$ \\ 'Department of Anesthesiology, Union \\ Hospital, Tongji Medical College, \\ Huazhong University of Science and \\ Technology, Wuhan, 430022, People's \\ Republic of China; ${ }^{2}$ Department of \\ Dermatology, Union Hospital, Tongji \\ Medical College, Huazhong University of \\ Science and Technology, Wuhan, 430022, \\ People's Republic of China
}

Background: The causal relationship between insomnia and migraine is contradictory and no study has been carried out among the Chinese population to date.

Methods: In this case, we conducted a case-control study and a bidirectional mendelian randomization (MR) analysis to determine whether insomnia is causally related to the development of migraine. The instrumental variables for insomnia were derived from the largest genome-wide association study of 1,331,010 participants, while the genetic instruments for migraine were available from the largest meta-analysis of migraine with 59,674 cases and 316,078 controls.

Results: In case-control study, subjects with insomnia have significantly higher risk of migraine ( $\mathrm{OR}=4.29$, 95\% CI: 3.21-5.74, $\mathrm{P}<0.001)$, compared with those without insomnia. The bidirectional two-sample MR analysis revealed that insomnia was significantly associated with higher risk of migraine $(\mathrm{OR}=1.24,95 \% \mathrm{CI}$ : $1.11-1.38, \mathrm{P}=1.01 \times 10-4)$, and the results were validated in the UK Biobank data. The results showed no indication for directional pleiotropy effects as assessed by the MR-Egger intercept $(\mathrm{P}>0.05)$.

Conclusion: Conclusively, our study highlighted that increased migraine risk was confined to subjects with a genetic pre-disposition to insomnia, and these findings had potential implications for improving the sleep quality to reduce the burden of migraine.

Keywords: mendelian randomization, insomnia, migraine, case-control

\section{Introduction}

Migraine, the most common headache disorder with a great impact on life quality of the patients, has become an important global public health concern. ${ }^{1-5}$ According to a systematic review and meta-analysis of community-based studies involving 6 million participants, migraine affects more than $10 \%$ of people worldwide. ${ }^{6}$ Meanwhile, unfavorable sleep disorders are also important public concern. The relationship between insomnia and migraine has been explored by many studies; however, the results were still inconsistent and the causality was undetermined to date. $^{7-10}$ This might be caused by potential residual confounding bias and reverse causality in epidemiologic studies. Besides, we also found that no study was conducted in Chinese population to date.

Mendelian randomization (MR), which utilizes genetic variants associated with exposure variables as genetic instruments to infer the causality, can be a useful tool for assessing the role of insomnia in migraine occurrence. ${ }^{11}$ The MR design is rarely affected by confounding bias and reverse causation due to the random
Correspondence: Jing Wu; Yue Qian Email tounj_002@I63.com; qianyue0II@sohu.com 
assortment of genetic variants at conception. ${ }^{12-14}$ It has been adopted in a wide range of diseases, including cardiovascular diseases, cancers, neuropsychiatric disorders, ischemic stroke, Type 2 diabetes, infectious diseases, and so on. ${ }^{15-26}$

In this case, we conducted a case-control study and a bidirectional MR analysis to determine whether insomnia is causally related to development of migraine. To ensure the authority and sophistication of the study, the instrumental variables for insomnia were derived from the largest genome-wide association study (GWAS) of 1,331,010 participants, while the genetic instruments for migraine were available from the largest meta-analysis of migraine with 59,674 cases and 316,078 controls.

\section{Patients and Methods}

\section{Study Subjects}

A diagnostic interview and a structured questionnaire were conducted based on the international classification of headache disorder diagnostic criteria, 3rd edition (ICHD-3). ${ }^{27}$ Controls did not suffer from migraine or other headache types. Subjects with severe systemic disease, tumor, major psychiatric disorder, or immunosuppressed patients were excluded. All the participants were recruited in Union Hospital and interviewed by headache specialist. Eligible participants were invited to complete a structured questionnaire. A total of 312 consecutive cases and 557 controls were contacted, and finally 300 migraine cases and 500 ageand gender-matched controls were included in this casecontrol study. The study received approval by the local Ethics Committee of the Union Hospital of Tongji Medical College and was conducted in accordance with the Declaration of Helsinki. All participants signed written informed consent prior to their enrolment to this study.

\section{Assessment of Insomnia}

According the method by UK Biobank study, ${ }^{28}$ insomnia complaints were assessed by asking: "Do you have trouble falling asleep at night or do you wake up in the middle of the night?" The participants were able to choose one of the following four answers: "never/rarely", "sometimes", or "usually". Insomnia cases were defined as participants who answered this question with "usually" or "sometimes", while participants answering "never/rarely" were defined as controls.

\section{Statistical Analysis and Mendelian Randomization}

The STATA 14.0 (Stata Corporation, College Station, TX, USA) was used for the statistical analyses, and a two-sided $\mathrm{P}$ value of $<0.05$ was considered statistically significant. The Chi-square $\left(\chi^{2}\right)$ test and Student's $t$-test were used to evaluate the distribution difference of selected variables in migraine cases and healthy controls. Adjusted odds ratios (ORs, adjusted for age, gender, family history of migraine, drinking status and BMI) and $95 \%$ confidence intervals (CIs) of the association between insomnia and migraine were calculated using unconditional logistic regressions.

Either insomnia is a cause or a downstream effect of migraine is still controversial, here we adopted a bidirectional $\mathrm{MR}$ design using $\mathrm{R}$ (version 3.6.0, $\mathrm{R}$ Foundation for Statistical Computing) packages and MRbase. ${ }^{29}$ The random-effect inverse-variance weighted (IVW) method was used as the primary analysis. Heterogeneity was estimated using Cochran's Q statistic. MR-Egger method was used for the detection of directional pleiotropic effects. The instrumental variables for insomnia were derived from the largest GWAS study to date of 1,331,010 participants, which identified 248 independent lead SNPs $\left(r^{2}<0.1\right)$ for insomnia ${ }^{28}$ Summary statistics data for migraine were available from finna-G6_MIGRAINE (3650 cases and 83,167 controls) and UK Biobank (ukb-b-16868, 13,597 cases and 449,336 controls). ${ }^{29}$ The genetic instruments for migraine were available from the largest meta-analysis of migraine with 59,674 cases and 316,078 controls. $^{30}$ Summary statistics data for insomnia were available from UK Biobank (ukbb-3957, with 462,341 participants).

\section{Results}

\section{Case-Control Study}

Table 1 presents the distributions of selected variables in migraine cases and healthy controls. We found no significant differences in distributions of age, gender and BMI. However, migraine cases have higher percentage of family history of migraine and drinking habits, compared with healthy controls. Table 2 shows the association between insomnia and migraine risk. Compared with subjects without insomnia, those with insomnia have significantly higher risk of migraine $(\mathrm{OR}=4.29,95 \%$ CI: $3.21-5.74$, $\mathrm{P}<0.001)$. Both those with insomnia sometimes $(\mathrm{OR}=3.51,95 \% \mathrm{CI}: 2.47-4.98, \mathrm{P}<0.001)$ and those with 
Table I Distributions of Selected Variables in Migraine Cases and Healthy Controls

\begin{tabular}{|l|c|c|c|}
\hline & $\begin{array}{c}\text { Cases } \\
(\mathbf{n = 3 0 0 )}\end{array}$ & $\begin{array}{c}\text { Controls } \\
(\mathbf{n = 5 0 0 )}\end{array}$ & P value \\
\hline Age & $35.7 \pm 7.6$ & $35.5 \pm 7.1$ & 0.707 \\
\hline $\begin{array}{c}\text { Gender } \\
\text { Male } \\
\text { Female }\end{array}$ & $37(12.3 \%)$ & $64(12.8 \%)$ & 0.847 \\
\hline $\begin{array}{l}\text { Family history of } \\
\text { migraine } \\
\text { Yes }\end{array}$ & $263(87.7 \%)$ & $436(87.2 \%)$ & \\
No & $207(69.0 \%)$ & $10 I(20.2 \%)$ & $<0.00$ I \\
\hline $\begin{array}{c}\text { Drinking status } \\
\text { Yes }\end{array}$ & $93(31.0 \%)$ & $399(79.8 \%)$ & \\
No & $203(67.7 \%)$ & 42 I (84.2\%) & \\
\hline BMl (Kg/m2) & $24.8 \pm 6.1$ & $24.1 \pm 5.2$ & 0.085 \\
\hline
\end{tabular}

Notes: $\mathrm{P}$ value in bold means statistically significant.

usual insomnia $(\mathrm{OR}=5.42,95 \% \mathrm{CI}: 3.78-7.76, \mathrm{P}<0.001)$ have higher risk of migraine.

\section{Genetically Predicted Insomnia on Migraine Risk}

Supplementary Table 1 listed the independent SNPs, which were used as the genetic instrumental variables for insomnia. We first evaluated the effect of genetically predicted insomnia on migraine risk in finna-G6_MIGRAINE. Insomnia was significantly associated with higher risk of migraine (Figure 1, $\left.\mathrm{OR}=1.24,95 \% \mathrm{CI}: 1.11-1.38, \mathrm{P}=1.01 \times 10^{-4}\right)$. The results were validated in the UK Biobank data (Figure 2). The results showed no indication for directional pleiotropy effects as assessed by the MR-Egger intercept $(\mathrm{P}>0.05)$. However, sensitivity analyses by MR-Egger did not validate the findings of IVW. Supplementary Figure 1 and $\underline{3}$ presented the forest plot, while Supplementary $\underline{\text { Figure } 2}$ and $\underline{4}$ presented the funnel plot.

\section{Genetically Predicted Migraine on Insomnia Risk}

The genetic instruments for migraine are listed in Supplementary Table 2. Using the IVW method, we did not detect significant causal relationship between genetically predicted migraine and insomnia risk $(\mathrm{OR}=1.01$, 95\% CI: $1.00-1.02, \mathrm{P}=0.159)$. The results also showed no indication for directional pleiotropy effects as assessed by the MR-Egger intercept $(\mathrm{P}>0.05)$.

\section{Discussion}

The current study explored the association between insomnia and migraine risk using a hospital-based case-control study in a Chinese population. We found that insomnia was significantly associated with higher risk of migraine, in both patients with insomnia sometimes and those with usual insomnia. Additionally, to remove the possible residual confounding bias and reverse causation, a bidirectional two-sample MR analysis was conducted and revealed that insomnia was causally related to the development of migraine. Collectively, our study highlights insomnia as a potentially modifiable risk factor for migraine.

Migraine ranks the second most disabling condition worldwide and has been revealed to be associated with cerebrovascular diseases, multiple sclerosis, depression, as well as sleep disorders. ${ }^{5,31-33}$ Although the association between migraine and sleep disorders is underlined by evidence from epidemiological studies, their relationship (causality or co-existence) has been the subject of much debate. According to a systematic review, ten association studies have evaluated the association between migraine and insomnia; however, their causal relationship is contradictory and none of the studies have been carried out among the Chinese. ${ }^{7}$ MR analyses have identified the causality between serum calcium, smoking, coronary artery disease and atrial fibrillation, Alzheimer's disease, intelligence, brain volume, sleep disturbances, blood pressure, social and socioeconomic outcomes and migraine. ${ }^{34-40}$ In this context, we conducted

Table 2 A Case-Control Study of Association Between Insomnia and Migraine Risk

\begin{tabular}{|l|c|c|c|c|}
\hline & Cases (n=300) & Controls (n=500) & OR (95\% CI)* & P \\
\hline No insomnia & 111 & 354 & 1.00 (reference) & \\
Insomnia & 189 & 146 & $4.29(3.21-5.74)$ & $<0.00 I$ \\
Sometimes & 91 & 86 & $3.51(2.47-4.98)$ & $<0.001$ \\
Usually & 98 & 60 & $5.42(3.78-7.76)$ & $<0.001$ \\
\hline
\end{tabular}

Notes: *Adjusted for age, gender, family history of migraine, drinking status and BMI. P value in bold means statistically significant. 


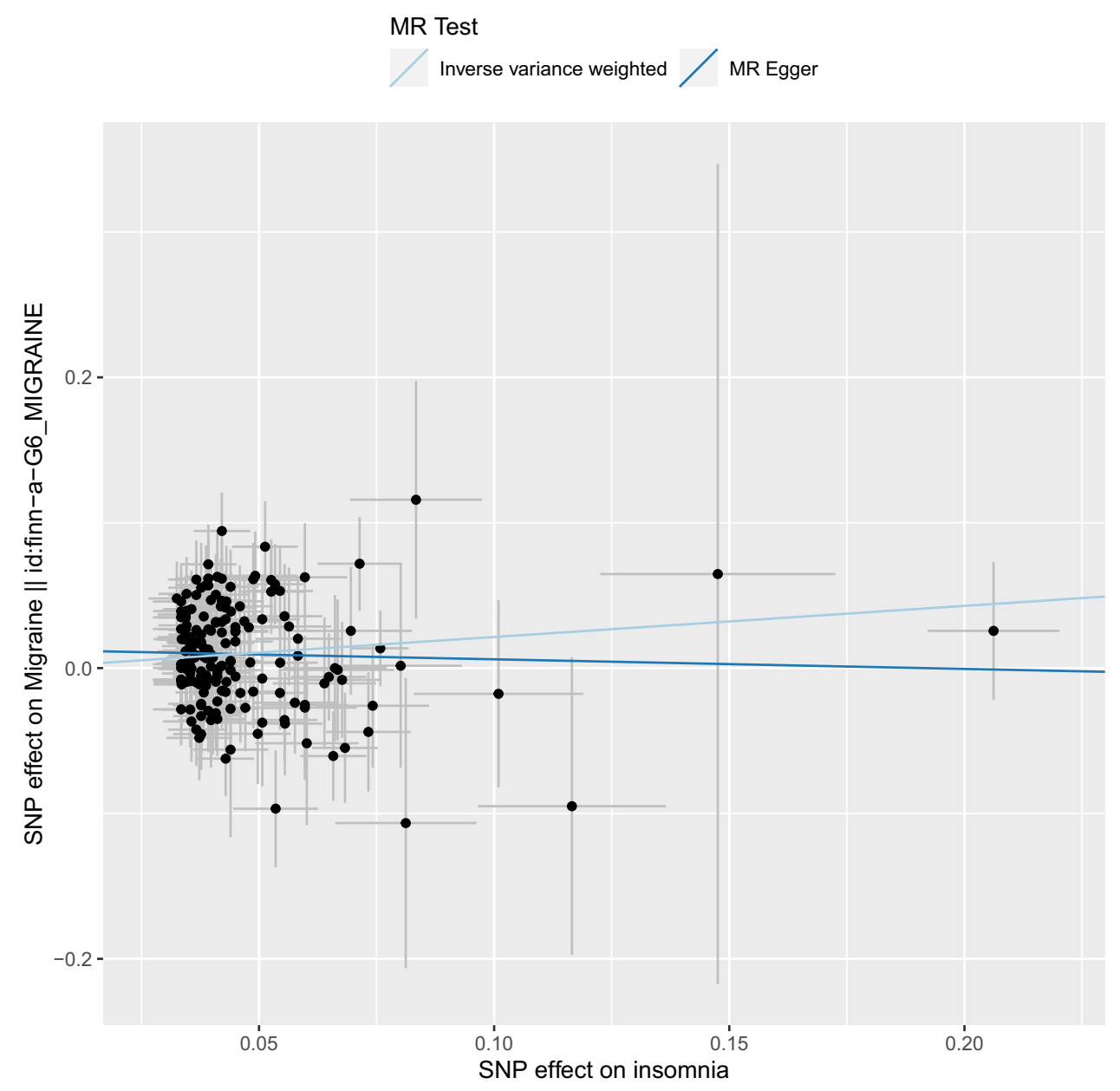

Figure I Scatter plots depicting the genetic association between insomnia and migraine risk in finn-a-G6_MIGRAINE.

this case-control study in Chinese and bidirectional twosample MR analysis. Our findings provides a direct evidence for a causal relationship between insomnia and occurrence of the migraine.

Our study has several methodological strengths. First, the joint application of epidemiological study and MR analysis was used to sort out the relationship between insomnia and migraine. Second, in this bidirectional twosample MR analysis, the instrumental variables for insomnia and migraine were derived from either the largest GWAS study of insomnia, or the largest meta-analysis of migraine. This ensured the authority and sophistication of the study. Third, the sample sizes in both case-control study and MR analysis maximize the statistical power for drawing the corresponding conclusions.

Several limitations should also be considered when interpreting the results. First, we adopted self-reported insomnia complaints rather than objective measures in the case-control study, due to the complexity of trait measurement. Second, collider bias remains an issue in MR analysis, and the possible pleiotropy effect might be concealed by small sample size. Third, due to the limitation of the GWAS summary statistics data, we did not perform stratified analyses on the major subtypes of migraine. Fourth, overlapping samples in the two-sample MR analysis may cause the results to be overestimated. Future larger studies upon migraine subtypes are needed.

\section{Conclusions}

In conclusion, we observed that insomnia was significantly associated with higher risk of migraine. Both epidemiological study and MR analysis suggested that increased migraine risk was confined to subjects with a genetic predisposition to insomnia. Further research should be directed towards exploring the molecular and cellular mechanisms underlying the effect of insomnia on increasing the 


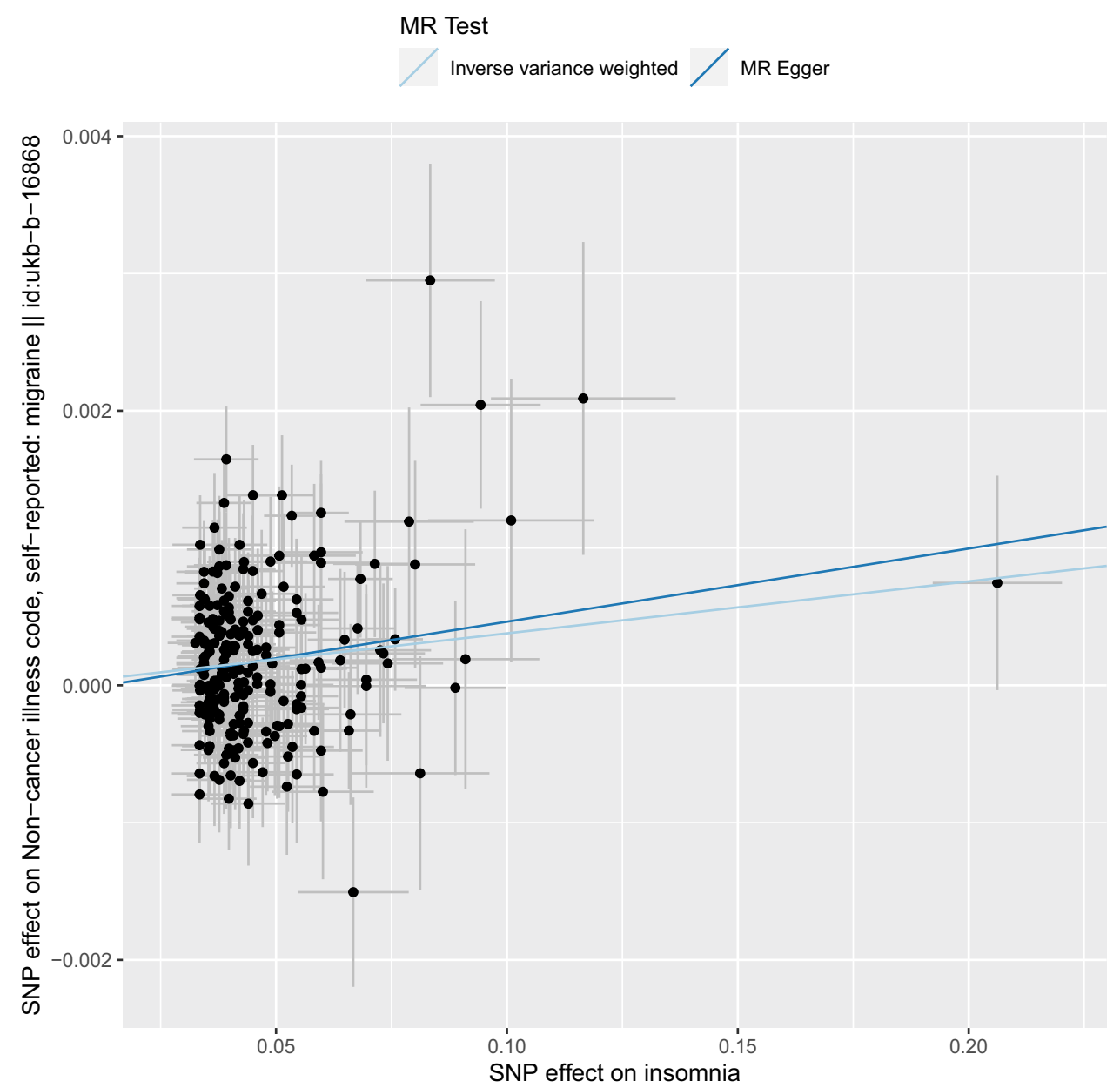

Figure 2 Scatter plots depicting the genetic association between insomnia and migraine risk in UK Biobank.

likelihood of migraine. Nonetheless, these findings have potential implications for improving the sleep quality to reduce the burden of migraine.

\section{Disclosure}

The authors declare that they have no conflict of interest.

\section{References}

1. El-Metwally A, Toivola P, AlAhmary K, et al. The epidemiology of migraine headache in Arab countries: a systematic review. ScientificWorldJournal. 2020;2020:4790254. doi:10.1155/2020/ 4790254

2. Navarro-Perez MP, Marin-Gracia M, Bellosta-Diago E, SantosLasaosa S. [Epidemiology of migraine in Spain and Latin America] Rev Neurol. 2020;71(3):110-118. Spanish.

3. Mattiuzzi C, Lippi G. Updates on migraine epidemiology. Eur J Neurol. 2020;27(3):e13. doi:10.1111/ene.14120

4. Marconi E, Pecchioli S, Nica M, et al. Epidemiology and determinants of chronic migraine: a real-world cohort study, with nested case-control analysis, in primary care in Italy. Cephalalgia. 2020;40 (5):461-469. doi:10.1177/0333102419889351
5. Burch RC, Buse DC, Lipton RB. Migraine: epidemiology, burden, and comorbidity. Neurol Clin. 2019;37(4):631-649. doi:10.1016/j. ncl.2019.06.001

6. Woldeamanuel YW, Cowan RP. Migraine affects 1 in 10 people worldwide featuring recent rise: a systematic review and meta-analysis of community-based studies involving 6 million participants. J Neurol Sci. 2017;372:307-315. doi:10.1016/j.jns.2016.11.071

7. Tiseo C, Vacca A, Felbush A, et al. Migraine and sleep disorders: a systematic review. J Headache Pain. 2020;21(1):126. doi:10.1186/ s10194-020-01192-5

8. Buse DC, Rains JC, Pavlovic JM, et al. Sleep disorders among people with migraine: results from the chronic migraine epidemiology and outcomes (CaMEO) Study. Headache. 2019;59(1):32-45. doi: $10.1111 /$ head. 13435

9. Rains JC, Poceta JS, Penzien DB. Sleep and headaches. Curr Neurol Neurosci Rep. 2008;8(2):167-175. doi:10.1007/s11910-008-0027-9

10. Jennum P, Jensen R. Sleep and headache. Sleep Med Rev. 2002;6 (6):471-479. doi:10.1053/smrv.2001.0223

11. Palmer TM, Lawlor DA, Harbord RM, et al. Using multiple genetic variants as instrumental variables for modifiable risk factors. Stat Methods Med Res. 2012;21(3):223-242. doi:10.1177/ 0962280210394459

12. Li S, Xu Y, Zhang Y, et al. Mendelian randomization analyses of genetically predicted circulating levels of cytokines with risk of breast cancer. NPJ Precis Oncol. 2020;4:25. doi:10.1038/s41698020-00131-6 
13. Lim SB, Di Lee W, Vasudevan J, Lim WT, Lim CT. Liquid biopsy: one cell at a time. NPJ Precis Oncol. 2019;3:23. doi:10.1038/s41698019-0095-0

14. Khoo BL, Shang M, Ng CH, Lim CT, Chng WJ, Han J. Liquid biopsy for minimal residual disease detection in leukemia using a portable blast cell biochip. NPJ Precis Oncol. 2019;3:30. doi:10.1038/s41698019-0102-5

15. Maners J, Gill D, Pankratz N, et al. A Mendelian randomization of gamma' and total fibrinogen levels in relation to venous thromboembolism and ischemic stroke. Blood. 2020;136(26):3062-3069. doi:10.1182/blood.2019004781

16. Russ TC, Harris SE, Batty GD. RESEARCH LETTER: pulmonary function and risk of Alzheimer dementia: two-sample Mendelian randomization study. Chest. 2020.

17. Butler-Laporte G, Nakanishi T, Mooser V, et al. The effect of angiotensin-converting enzyme levels on Covid-19 susceptibility and severity: a Mendelian randomization study. Int J Epidemiol. 2020;50:75.

18. Peters TM, Holmes MV, Richards JB, et al. Sex differences in the risk of coronary heart disease associated with type 2 diabetes: a mendelian randomization analysis. Diabetes Care. 2020;44:556.

19. Rosoff DB, Davey Smith G, Mehta N, Clarke TK, Lohoff FW. Evaluating the relationship between alcohol consumption, tobacco use, and cardiovascular disease: a multivariable Mendelian randomization study. PLoS Med. 2020;17(12):e1003410. doi:10.1371/journal. pmed. 1003410

20. Larsson SC, Mason AM, Kar S, et al. Genetically proxied milk consumption and risk of colorectal, bladder, breast, and prostate cancer: a two-sample Mendelian randomization study. BMC Med. 2020;18(1):370. doi:10.1186/s12916-020-01839-9

21. Arvanitis M, Qi G, Bhatt DL, et al. A linear and non-linear mendelian randomization analysis of the association between diastolic blood pressure and cardiovascular events: the $\mathrm{j}$ curve revisited. Circulation. 2020;143:895.

22. Aung N, Sanghvi MM, Piechnik SK, Neubauer S, Munroe PB, Petersen SE. The effect of blood lipids on the left ventricle: a Mendelian Randomization Study. J Am Coll Cardiol. 2020;76 (21):2477-2488. doi:10.1016/j.jacc.2020.09.583

23. Rogne T, Solligard E, Burgess S, et al. Body mass index and risk of dying from a bloodstream infection: a Mendelian randomization study. PLoS Med. 2020;17(11):e1003413. doi:10.1371/journal. pmed.1003413

24. Dong $\mathrm{X}$, Zhu Z, Wei $\mathrm{Y}$, et al. Plasma insulin-like growth factor binding protein-7 (IGFBP-7) contributes causally to ARDS 28-day mortality: evidence from multi-stage Mendelian randomization. Chest. 2020;78:151.

25. Rosoff DB, Smith GD, Lohoff FW. Prescription opioid use and risk for major depressive disorder and anxiety and stress-related disorders: a multivariable mendelian randomization analysis. JAMA Psychiatry. 2020;78:151.

26. Wu F, Huang Y, Hu J, Shao Z. Mendelian randomization study of inflammatory bowel disease and bone mineral density. BMC Med. 2020;18(1):312. doi:10.1186/s12916-020-01778-5

Pharmacogenomics and Personalized Medicine

\section{Publish your work in this journal}

Pharmacogenomics and Personalized Medicine is an international, peer-reviewed, open access journal characterizing the influence of genotype on pharmacology leading to the development of personalized treatment programs and individualized drug selection for improved safety, efficacy and sustainability. This journal is indexed
27. Arnold M. Headache classification committee of the international headache society (IHS) The international classification of headache disorders. Cephalalgia. 2018;38(1):1-211.

28. Jansen PR, Watanabe K, Stringer S, et al. Genome-wide analysis of insomnia in 1,331,010 individuals identifies new risk loci and functional pathways. Nat Genet. 2019;51(3):394-403. doi:10.1038/ s41588-018-0333-3

29. Hemani G, Zheng J, Elsworth B, et al. The MR-Base platform supports systematic causal inference across the human phenome. eLife. 2018;5:7.

30. Gormley P, Anttila V, Winsvold BS, et al. Meta-analysis of 375,000 individuals identifies 38 susceptibility loci for migraine. Nat Genet. 2016;48(8):856-866. doi:10.1038/ng.3598

31. Gelfand AA, Gelfand JM, Goadsby PJ. Migraine and multiple sclerosis: epidemiology and approach to treatment. Mult Scler Relat Disord. 2013;2(2):73-79. doi:10.1016/j.msard.2012.10.003

32. Magalhaes JE, Sampaio Rocha-Filho PA. Migraine and cerebrovascular diseases: epidemiology, pathophysiological, and clinical considerations. Headache. 2018;58(8):1277-1286. doi:10.1111/ head. 13378

33. Yang Y, Ligthart L, Terwindt GM, Boomsma DI, RodriguezAcevedo AJ, Nyholt DR. Genetic epidemiology of migraine and depression. Cephalalgia. 2016;36(7):679-691. doi:10.1177/ 0333102416638520

34. Yin P, Anttila V, Siewert KM, Palotie A, Davey Smith G, Voight BF. Serum calcium and risk of migraine: a Mendelian randomization study. Hum Mol Genet. 2017;26(4):820-828.

35. Johnsen MB, Winsvold BS, Borte S, et al. The causal role of smoking on the risk of headache. A Mendelian randomization analysis in the HUNT study. Eur J Neurol. 2018;25(9):1148-e1102. doi:10.1111/ ene. 13675

36. Daghlas I, Guo Y, Chasman DI. Effect of genetic liability to migraine on coronary artery disease and atrial fibrillation: a Mendelian randomization study. Eur J Neurol. 2020;27(3):550-556. doi:10.1111/ ene. 14111

37. Daghlas I, Rist PM, Chasman DI. Effect of genetic liability to migraine on cognition and brain volume: a Mendelian randomization study. Cephalalgia. 2020;40(9):998-1002. doi:10.1177/ 0333102420916751

38. Guo Y, Rist PM, Daghlas I, et al. A genome-wide cross-phenotype meta-analysis of the association of blood pressure with migraine. Nat Commun. 2020;11(1):3368. doi:10.1038/s41467-020-17002-0

39. Daghlas I, Vgontzas A, Guo Y, Chasman DI. Habitual sleep disturbances and migraine: a Mendelian randomization study. Ann Clin Transl Neurol. 2020;7(12):2370-2380. doi:10.1002/acn3.51228

40. Harrison S, Davies AR, Dickson M, et al. The causal effects of health conditions and risk factors on social and socioeconomic outcomes: mendelian randomization in UK Biobank. Int J Epidemiol. 2020;49 (5):1661-1681. doi:10.1093/ije/dyaa114 on the American Chemical Society's Chemical Abstracts Service (CAS). The manuscript management system is completely online and includes a very quick and fair peer-review system, which is all easy to use. Visit http://www.dovepress.com/testimonials.php to read real quotes from published authors.

\section{Dovepress}

